Revue internationale P.M.E.

Économie et gestion de la petite et moyenne entreprise

\title{
L'évolution de la gestion de la qualité totale dans les petites entreprises sous-traitantes du secteur de l'aéronautique
}

\section{Emilio Esposito et Mario Raffa}

Volume 9, numéro 2, 1996

URI : https://id.erudit.org/iderudit/1008262ar

DOI : https://doi.org/10.7202/1008262ar

Aller au sommaire du numéro

Éditeur(s)

Presses de l’Université du Québec

ISSN

0776-5436 (imprimé)

1918-9699 (numérique)

Découvrir la revue

Citer cet article

Esposito, E. \& Raffa, M. (1996). L'évolution de la gestion de la qualité totale dans les petites entreprises sous-traitantes du secteur de l'aéronautique. Revue internationale P.M.E., 9(2), 57-80. https://doi.org/10.7202/1008262ar
Résumé de l'article

Ce travail met en évidence l'évolution de la gestion de la qualité totale dans les petites et moyennes entreprises sous-traitantes du secteur de l'aéronautique dans deux réalités industrielles différentes, en France et en Italie. L'enquête sur le terrain, qui s'est déroulée en Italie (Campanie) et en France (région lyonnaise), montre que le niveau d'attention aux problématiques de la qualité est élevé dans les deux pays. Toutefois, le degré de diffusion de la philosophie de gestion de la qualité totale dans les entreprises françaises est légèrement plus élevé que dans le cas des entreprises italiennes. 


\title{
L'évolution de la gestion de la qualité totale dans les petites entreprises sous-traitantes du secteur de l'aéronautique
}

Emilio ESPOSITO

Mario RAFFA

ODISSEO-DIS, Université de Naples

MOTS CLÉS
Sous-traitance - Secteur de l'aéronautique - France - Italie Parc machines - Nouvelles technologies - Formation Gestion de la qualité totale

\begin{abstract}
RÉSUMÉ
Ce travail met en évidence l'évolution de la gestion de la qualité totale dans les petites et moyennes entreprises sous-traitantes du secteur de l'aéronautique dans deux réalités industrielles différentes, en France et en Italie. L'enquête sur le terrain, qui s'est déroulée en Italie (Campanie) et en France (région lyonnaise), montre que le niveau d'attention aux problématiques de la qualité est élevé dans les deux pays. Toutefois, le degré de diffusion de la philosophie

\section{LES AUTEURS}

Emilio Esposito est enseignant en économie et organisation de l'entreprise à la Faculté d'ingénierie de l'Université de Naples "Federico II".

Mario Raffa est enseignant en économie et organisation de l'entreprise auprès de la Faculté d'ingénierie de l'Université de Naples "Federico II ". II est aussi le responsable scientifique de ODISSEO (Observatoire sur l'organisation et l'innovation technologique) et président du cours de maîtrise en ingénierie gestionnaire. Adresse: ODISSEO, Università degli studi di Napoli “ Federico II ", Dipartimento di informatica e sistemistica, Via Diocleziano 328, 80124 Naples, Italie.

Ce travail a été réalisé dans le cadre du projet stratégique CNR "Transfert des technologies des projets finalisés". Bien que ce travail soit le fruit d'une activité de recherche commune, la rédaction actuelle a été préparée par Emilio Esposito.
\end{abstract}


de gestion de la qualité totale dans les entreprises françaises est légèrement plus élevé que dans le cas des entreprises italiennes.

\begin{abstract}
This work shows the evolution of Total Quality Management (TQM) in small subcontracting firms in the aircraft industry in two different industrial situations; in Italy and France. Field research carried out in Campania in Italy and in the Lyon's area of France shows that in both situations the attention given to quality problems is high but there are managerial differences that create a different orientation towards human resources training and quality problems. Among Italian firms, TQM still predominantly regard the area of production, a sign of a still traditional approach, which is above all concerned with respecting the technical specifications of the customer. On the other hand, French firms are clearly oriented towards TQM, where quality concerns all areas of a firm's activities; from production to administration up to management.
\end{abstract}

\title{
RESUMEN
}

Este trabajo pone en evidencia la evolución del Total Quality Management en las pequeñas y medianas empresas subproveedoras del sector aeronáutico, en dos diferentes realidades industriales, la francesa y la italiana. El studio sobra el campo realizado en Italia en Campania y en Francia en el área de Lión, muestra que en ambas realidades el nivel de atención hacia las problemáticas de la calidad es elevado, pero que sin embargo existen diversidades de gestión que determinan una diversa orientatión de la empresa hacia la formación de los recursos humanos y las problemáticas de la calidad. Entre las empresas italianas, la gestión de la calidad esta todavía con preponderancia dirigida hacia el ária de la producción, señal evidente de una visión aún de tipo tradicional, atenta, por lo tanto, sobre todo al respeto de las específicas técnicas del cliente. Las empresas francesas, en cambio, están orientadas hacia el Total Quality Management; la calidad interesa a todas las áreas de la empresa, desde la producción pasando por el área administrativa, hasta la directiva.

\section{Introduction}

Ce travail fait partie d'un filon de recherche pluriennale dont l'objectif général est l'étude de l'évolution des rapports de sous-traitance dans les secteurs à haute technologie des principaux pays industrialisés. Lors d'un travail précédent, l'analyse d'un exemple avait pour but d'évaluer le degré de circulation de la technologie entre les entreprises principales et les entreprises «fournisseurs », de même que les résultats empiriques relevés (Esposito et Raffa, 1992).

On a voulu vérifier dans cette étude si le niveau de la gestion de la qualité totale dans les entreprises qui fournissent des activités de sous-traitance est influencé par des caractéristiques nationales qui conditionneraient les compor- 
tements et les stratégies des entreprises. Pour la gestion de la qualité totale, on entend, selon les normes ISO, une prise de contact avec la gestion des entreprises basée sur la participation de tout le personnel et visant la rentabilité à long terme à travers la satisfaction du client.

Cet aspect est important parce qu'il permet de distinguer les tendances générales qui caractérisent l'évolution des rapports de sous-traitance de celles qui dépendent, au contraire, de conditions particulières liées aux différentes localisations géographiques. C'est dans ce but qu'une comparaison a été faite entre les petites et moyennes entreprises travaillant en sous-traitance dans le secteur de l'aéronautique en Italie et en France.

D'autres recherches effectuées ces dernières années ont déjà mis en évidence l'importance des méthodes de sous-traitance pour assurer un avantage compétitif aux entreprises des pays qui les ont adoptées. Il a été mis en évidence, en particulier, que l'organisation de «classe mondiale » dépend de manière significative des fournisseurs directs et indirects. En outre, ces recherches ont souligné l'importance de la qualité ou, mieux encore, du système global de la gestion de la qualité (gestion de la qualité totale) pour assurer la réalisation et le maintien des niveaux d'excellence (Hines, 1994). raisons :

Le choix du secteur de l'aéronautique est significatif pour plusieurs

1. Le secteur de l'aéronautique est représentatif des industries à haute technologie puisque le taux de la dépense pour la recherche et le développement atteint en moyenne $20 \%$ du chiffre d'affaires, un pourcentage supérieur à celui de tout autre secteur industriel, y compris les secteurs de l'électronique et de la pharmaceutique (OCDE, 1991 ; Tyson, 1992).

2. Les entreprises du secteur opèrent depuis toujours avec des normes de qualité et de fiabilité qui n'ont pas leur pareil dans d'autres industries. Cela est la conséquence du degré élevé de qualité auquel sont soumises les lignes aériennes et des normes européennes contraignantes auxquelles les entreprises productrices d'avions doivent se conformer pour obtenir la certification du produit.

3. Depuis toujours dans le secteur de l'aéronautique, les rapports entre donneurs d'ordres et sous-traitants sont réglés dans le but d'assurer des normes de qualité élevées du produit. Cela a facilité le développement, avec de nombreuses années d'avance par rapport à d'autres industries, de certaines caractéristiques des relations de sous-traitance basées sur l'échange de connaissances et sur la confiance réciproque (De Maio et Maggiore, 1992 ; Mowery, 1991 ; Sako, 1992). 
La comparaison entre la réalité italienne et française est significative dans la mesure où ces deux réalités géographiques synthétisent bien les différences entre les divers pays européens.

1. Il existe de profondes différences structurelles entre l'industrie aéronautique française et l'industrie aéronautique italienne. La première a une dimension et des capacités technologiques plus importantes; elle est liée par des rapports de collaboration étroits avec les autres entreprises aéronautiques européennes; elle a un rôle de leader au niveau mondial (CEE, 1993 ; Moreau, 1985). La seconde a une faible dimension et elle est liée aux principales entreprises des États-Unis (Boeing, McDonnell Douglas) par des rapports de «collaboration asymétrique » (CEE, 1993 ; Jane's, 1994).

2. Déjà dans les années 1970, l'activité de sous-traitance a eu un rôle important dans le système économique français, comme en témoignent les nombreuses publications scientifiques sur le sujet ${ }^{1}$ (Sallez et Schlegel, 1963 ; Chaillou, 1977 ; Barthomieu et al., 1983 ; Vennin, 1975). En Italie, par contre, le phénomène de la décentralisation productive dans les années 1970 apparaissait comme une conséquence nécessaire et structurelle du développement économique du pays liée à la faible capacité d'innovation des grandes entreprises ${ }^{2}$.

3. Les activités des entreprises sous-traitantes italiennes sont surtout orientées vers des commandes nationales, tandis qu'en France, ces entreprises ont une forte propension à se diriger vers les marchés internationaux, aidées en cela par les donneurs d'ordres et par les normes ${ }^{3}$.

1. La création des premières bourses pour la sous-traitance remonte en France au début des années 1960, et dès 1970, des organismes comme le CENAST (Centre national de la sous-traitance) et l'INSTAT (Institut national pour la sous-traitance et l'assistance technique) ont été créés; ils ont pour mission de coordonner et de soutenir les actions de promotion pour la sous-traitance et «l'enseignement d'un langage commun » aux commettants et aux sous-traitants. En décembre 1975, la loi sur la sous-traitance a été approuvée, faisant de la France l'unique pays européen a être doté d'un dispositif de protection particulier en faveur des sous-traitants (Altersohn, 1992).

2. Dans ces années-là, les entreprises italiennes de grandes dimensions qui devaient affronter une réalité de forte rigidité du travail, au lieu de se tourner vers l'innovation de l'appareil industriel, tentèrent, à travers la décentralisation, de contourner l'obstacle de la rigidité du travail en utilisant les différences salariales par rapport aux petites entreprises.

3. En effet, en France, dès 1970, des « services de promotion consulaires » ont été institués; ce sont des dispositifs d'assistance technico-commerciales en faveur des PME dans le cadre des activités des chambres de commerce régionales et locales. 
Dans les pages qui suivent, on verra comment ces homogénéités et ces diversités conditionnent les entreprises de sous-traitance dans leurs rapports avec les donneurs d'ordres et dans la propension à l'innovation et à la diffusion de la gestion de la qualité totale.

\section{Méthodologie de recherche et échantillonnage des entreprises étudiées}

Cette recherche se base sur les résultats d'une enquête sur le terrain que l'Observatoire ODISSEO-DIS a réalisé lui-même auprès de 38 petites et moyennes entreprises italiennes et françaises qui pratiquent des activités de sous-traitance dans le secteur de l'aéronautique.

Les différentes phases de la recherche sont illustrées à la figure 1. On a effectué l'enquête avec un questionnaire semi-structuré. Tous les éléments ont été réunis et mis à jour par des entrevues à des nombreux dirigeants d'entreprises et par plusieurs rencontres et visites des entreprises. Dans cet article, on a utilisé seulement quelques éléments des différentes sections qui constituent le questionnaire.

On a interrogé les plus hauts dirigeants de 18 entreprises de sous-traitance françaises et de 20 entreprises italiennes. Les 18 entreprises françaises sont situées essentiellement (12 sur 18) dans la région Rhône-Alpes (seconde région française pour le nombre de sous-traitants), le reste est réparti dans deux autres régions : Île-de-France et Midi-Pyrénées. Ces entreprises soustraitantes réalisent des parties, des sous-ensembles et des ensembles finis pour différentes grandes entreprises aéronautiques françaises (Aérospatiale, Dassault, Snecma, Thomson, Airbus industrie) qui, tout en opérant dans divers services du secteur (cellule, propulseur et systèmes), ont en commun le fait de développer des produits répondant à des normes de qualité et de fiabilité élevées.

Les 20 entreprises italiennes sont situées dans une seule région (La Campanie) où se trouvent aussi les trois principales entreprises donneurs d'ordres du secteur de l'aéronautique (Alénia, Alfa Romeo Avio et Magnaghi) autour desquelles gravitent la plupart d'entre elles. Cette limitation régionale n'invalide pas les résultats de la recherche. En effet, bien que la sous-traitance concernant le secteur de l'aéronautique soit essentiellement concentrée dans deux régions (Campanie et Piémont), il n'existe pas de différences substantielles dans le comportement des entreprises de sous-traitance entre les deux zones (Esposito et Raffa, 1994).

La totalité de l'échantillonnage est constituée de petites et de moyennes entreprises. Au total, cinq entreprises ont plus de 100 employés, cinq appartiennent à la catégorie qui comprend entre 50 et 99 employés, vingt font partie 


\section{FIGURE 1}

\section{Les différentes phases de la recherche}

Définition des objectifs et du plan de recherche.

Préparation et expérimentation du questionnaire semi-structuré.

L'an 1986.

10 entrevues avec plusieurs dirigeants d'entreprises sous-traitantes.

Les auteurs connaissaient déjà les caractéristiques des entreprises commettantes qui venaient d'une recherche commencée en 1979.

$\downarrow$

Questionnaire définitif.

Choix d'un échantillon italien des entreprises sous-traitantes et commettantes.

Élaboration «évolutive» des éléments.

Publication des premiers résultats.

Les années 1987-1990.

$\downarrow$

60 entrevues avec des entreprises sous-traitantes. Pour plusieurs entreprises, les rencontres et les entrevues ont été répétées tous les deux ans.

$\downarrow$

Poursuite de la recherche avec le questionnaire de base.

Élaboration de questionnaires additionnels pour étudier les problèmes

soulevés pendant la recherche qui deviennent de plus en plus importants

dans le débat scientifique. Le modèle de diffusion de la technologie, utilisé pour mesurer les voies de connexion parmi les entreprises commettantes

et les entreprises sous-traitantes a valu le «Prix d'excellence» pour la meilleure communication par le ICSB

(International Council for Small Business).

Élaboration «évolutive» des éléments.

Les années 1991-1992.

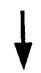

100 entrevues avec des entreprises sous-traitantes ;

pour 78 d'entre elles, on a utilisé le même questionnaire.

$\downarrow$

La recherche est étendue à d'autres pays européens.

La mise à jour des éléments concernant les entreprises italiennes continue.

Les années 1993-1994.<smiles>C1CC1</smiles>

25 entrevues avec des entreprises sous-fournisseuses françaises dont les commettants présentent des analogies avec les commettants italiens. 
de la catégorie qui comprend entre 20 et 49 unités, huit ont moins de 19 employés (figure 2). La catégorie la plus représentée est celle dont le nombre d'employés est compris entre 20 et $49^{4}$.

FIGURE 2

Répartition des entreprises interrogées par nombre d'employés

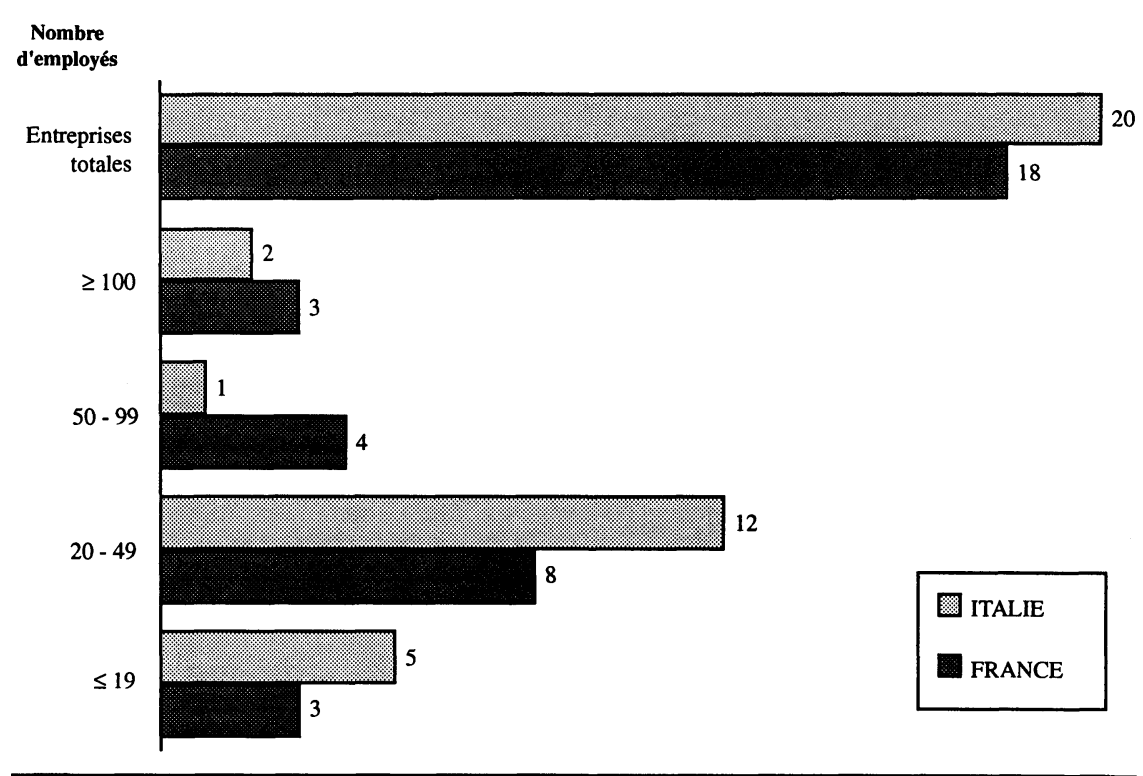

Le système de la sous-traitance aéronautique est donc caractérisé, aussi bien en France qu'en Italie, par des entreprises de petites dimensions qui, en moyenne, ont moins de 100 employés 5 .

En ce qui concerne les activités de production, les entreprises de l'échantillonnage présentent une forte homogénéité, aussi bien en ce qui concerne les activités plus traditionnelles (mécanique de précision pour l'aéronautique,

4. Du point de vue des dimensions, il existe une forte homogénéité entre l'échantillonnage d'entreprises françaises et italiennes. Pour une sécurité majeure, nous avons utilisé le test statistique de Wilcoxon; le résultat est amplement inférieur à la valeur critique de $\mathrm{P}=5 \%$. Nous pouvons donc réfuter l'hypothèse de diversité entre les entreprises françaises et italiennes.

5. Comme donnée comparative, on peut penser au cas de la sous-traitance dans le secteur de la construction automobile. En Italie, les principales entreprises soustraitantes ont une dimension moyenne d'environ 350 employés (Esposito et Raffa, 1994). 
travail de la tôle, etc.) que les activités représentatives des nouvelles dynamiques de la sous-traitance (travail de matériaux composites, assemblage spécialisé, réalisation d'équipements).

En conclusion, l'échantillonnage d'entreprises sous-traitantes est homogène par la dimension, les activités de production et les caractéristiques technologiques du secteur auquel s'adresse la sous-traitance. Toutefois, ces entreprises opèrent dans des cadres présentant des différences qui découlent des caractéristiques des donneurs d'ordres et du degré d'attention porté aux thématiques de la qualité dans leur pays respectif.

\section{Quelques caractéristiques structurelles de la sous-traitance dans le secteur de l'aéronautique dans les deux pays}

Après avoir brièvement présenté la méthodologie de la recherche et l'échantillonnage des entreprises sous-traitantes, nous montrerons, dans ce paragraphe, comment l'histoire du secteur et les caractéristiques des principales entreprises productrices d'avions ont une répercussion sur les rapports de la sous-traitance.

Du point de vue technologique, l'industrie aéronautique française se présente comme une des industries de pointe sur le plan mondial, bénéficiant d'un grand prestige international, aussi bien dans le secteur civil que militaire. Les entreprises françaises conçoivent et réalisent depuis toujours des appareils complets, seules ou en coopération avec d'autres grandes entreprises européennes : Dassault, par exemple, qui produit seule des avions de combat militaires (la famille Mirage), est depuis longtemps un leader mondial; l'Aérospatiale réalise seule des hélicoptères et occupe une position de leader dans le secteur du transport aérien à travers le groupe Airbus (avec la British Aerospace, l'allemande DASA et l'espagnole CASA) et le groupe ATR (avec Alenia); la SNECMA est la partie stable de l'oligopole mondial du secteur des propulseurs aéronautiques. La grande capacité technologique et productive de l'industrie aéronautique française a eu comme retombée géographique le développement, dès les années $1960^{6}$, d'un réseau d'entreprises de soustraitance qui, avec le temps, s'est de plus en plus consolidé.

L'industrie aéronautique italienne voit, elle aussi, une forte présence d'entreprises de grandes traditions qui, après la guerre, ont dû supporter de

6. Par l'avènement de la propulsion à jet dans le transport aérien, l'industrie aéronautique opère un saut de qualité considérable et elle est appelée à répondre à une demande croissante d'appareils de transport. C'est dans ces années que les principales entreprises aéronautiques, à l'échelle mondiale, consolident leur propre réseau d'entreprises sous-traitantes sur les plans national et international. 
grandes difficultés accompagnées d'une perte de compétitivité sur le plan international; elles se sont vues reléguées à un rôle secondaire par rapport aux principales entreprises de l'oligopole mondial. Avant 1965, en Italie, il n'existait aucune entreprise de grandes dimensions opérant dans le secteur du transport aérien. L'unique société du secteur, l'Aefer, s'occupait essentiellement d'activités de sous-traitance pour les grandes entreprises des États-Unis. Ce n'est qu'à la fin des années 1970, à la suite de la réorganisation du secteur de l'aéronautique italien que naquit l'Aeritalia $^{7}$ qui très vite devint la principale société italienne du secteur. Dans les années 1970, l'Aeritalia commença à s'insérer, même si en position minoritaire, dans l'oligopole international et à développer son propre réseau de petites et moyennes entreprises sous-traitantes ${ }^{8}$.

L'industrie aéronautique française a donc développé son réseau d'entreprises sous-traitantes avec plus d'une dizaine d'années d'avance par rapport à l'aéronautique italienne.

L'enquête sur le terrain, qui confirme ce que nous venons d'illustrer, met en évidence que les entreprises sous-traitantes françaises sont présentes sur le marché depuis plus longtemps que les entreprises italiennes. La moitié des compagnies françaises sont nées avant 1965, tandis que pour la plus grande partie des entreprises italiennes ( $70 \%$ environ), la date de naissance se situe entre 1966 et 1975 (figure 3).

En 1991, le chiffre d'affaires de l'aéronautique française atteignait 17,4 milliards d'ECU contre un peu plus de 5 milliards pour le chiffre d'affaires italien; toujours en 1991, l'industrie française employait 118 mille personnes environ contre 47 mille en Italie (CEE, 1993). Le rapport chiffre d'affaires/personnel de l'industrie française était de 149 mille ECU contre

7. L'Aeritalia est née de la participation de l'Aerfer (entreprise du groupe IRIFinmeccanica) et de la Fiat Aviation. Cette tentative procédait du besoin de réorganiser le secteur de l'aéronautique italien qui voyait la présence de nombreuses entreprises en concurrence entre elles et incapables de rivaliser avec les grandes entreprises sur le plan international. Au début des années 1990, l'Aeritalia et la Selenia (Société opérant dans le secteur des missiles, de l'électronique/ radar et de l'espace) donnent vie au groupe actuel Alenia. L'Alenia opère dans divers secteurs : aéronautique (civile et militaire), missile, radar, espace. Elle est présente dans le secteur de l'aéronautique aussi bien avec des produits réalisés en coopération ATR (avec la française Aérospatiale), AMX (avec la Aermacchi et la brésilienne Embraer), Tornado-MRCA (avec l'espagnole CASA, British Aerospace et l'allemande DASA), qu'avec une activité de sous-traitance internationale.

8. Toutefois, aujourd'hui encore, une partie importante du chiffre d'affaires de l'aéronautique des principales entreprises italiennes est réalisée avec la soustraitance internationale pour les compagnies américaines Boeing et McDonnell Douglas. 


\section{FIGURE 3}

Répartition des entreprises interrogées par année de naissance

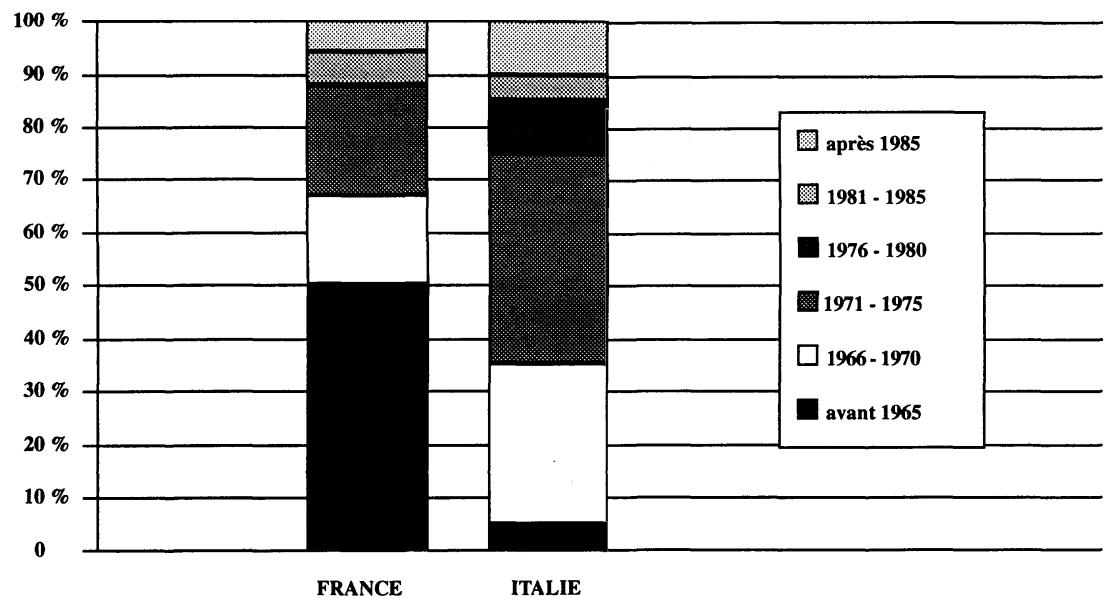

108 mille pour l'industrie italienne. L'Aérospatiale, la principale entreprise française, présentait un rapport chiffre d'affaires / personnel d'environ 270 mille ECU contre 104 mille pour l'Alenia, la plus importante entreprise italienne.

Les raisons de cet écart dans le rapport chiffre d'affaires / personnel doivent être recherchées dans l'organisation différente des activités productives. Les entreprises aéronautiques françaises confient davantage de travail à l'extérieur, par rapport à leurs correspondantes italiennes. En effet, elles conçoivent et réalisent complètement l'avion, elles sont en relation avec de nombreuses autres entreprises pour l'achat des propulseurs, des équipements, mais aussi d'éléments et de composants de la structure de l'avion 9 . Par contre, la principale entreprise italienne du secteur réalise elle-même des activités de soustraitance pour les grandes entreprises des États-Unis et d'Europe.

Le rôle différent des principales entreprises aéronautiques sur la scène mondiale a des retombées sur les activités de sous-traitance. Les entreprises sous-traitantes françaises, grâce à leurs donneurs d'ordres qui opèrent directement sur les marchés internationaux, sont entrées depuis longtemps

9. La réalisation de l'avion naît de la confluence d'éléments et de composants qui proviennent de trois sous-secteurs principaux : la cellule, le propulseur et les équipements. L'entreprise qui conçoit l'avion (entreprise leader) appartient au domaine de la cellule. Si l'on considère que l'importance de ce sous-secteur sur les coûts globaux de l'avion est d'environ $50 \%$ et que l'entreprise leader confie à l'extérieur une partie notable de ces activités, on peut en déduire que l'entreprise qui conçoit l'avion ne réalise que 30-35\% (en termes de coût) de l'avion. 
dans un système compétitif sur une échelle européenne et mondiale ${ }^{10}$. Cet aspect est clairement démontré par une forte propension à l'exportation de la part des entreprises sous-traitantes françaises (environ $18 \%$ du chiffre d'affaires) par rapport aux entreprises italiennes correspondantes (un peu moins de $9 \%$ ) (figure 4).

FIGURE 4

\section{Localisation géographique du marché des entreprises interrogées}

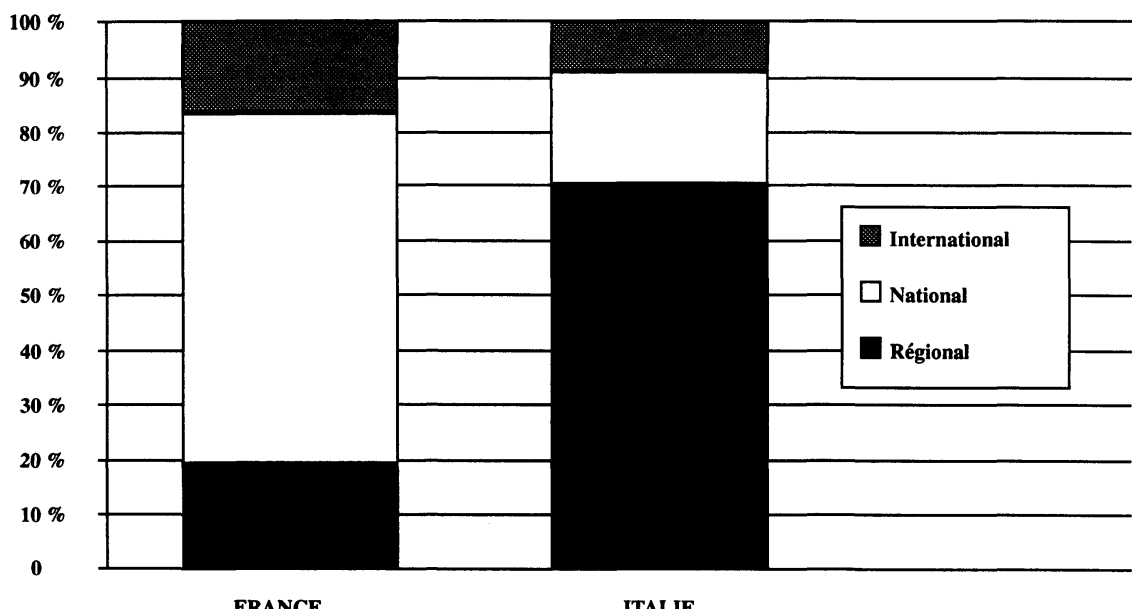

Pour pouvoir opérer avec plusieurs grands donneurs d'ordres nationaux, les entreprises sous-traitantes françaises ont dirigé leurs efforts vers une politique de diversification du donneur d'ordre. Les deux premiers donneurs d'ordres procurent environ $40 \%$ du chiffre d'affaires des entreprises sous-traitantes françaises (figure 5).

L'avantage pour les entreprises sous-traitantes françaises est indiscutable ; celles-ci, en vertu d'une diversification plus importante, ont une faible dépendance à l'égard du donneur d'ordre principal ${ }^{11}$, elles peuvent donc mieux réagir aux conjonctures négatives d'un client en déplaçant, au besoin,

10. Par exemple, les entreprises sous-traitantes de l'Aérospatiale qui interviennent dans la réalisation d'éléments et de composants pour le consortium Airbus ont eu la possibilité de s'insérer dans un réseau international de sous-traitance qui voit la présence de British Aerospace, de la CASA et de la DASA, mais aussi de la Fokker qui y participe comme membre associé du consortium.

11. Le poids du commettant sur le chiffre d'affaires du sous-traitant est indicatif du degré de dépendance du sous-traitant envers le commettant (Scott et Westbrook, 1991). 
son activité vers d'autres donneurs d'ordres, aussi bien sur le plan national qu'international.

La situation des entreprises sous-traitantes italiennes est différente : presque $78 \%$ de leur chiffre d'affaires est réalisé pour les deux premiers donneurs d'ordres. Cela indique une position de forte dépendance et explique pourquoi les sous-traitants italiens, incapables de se détacher rapidement de l'Alenia, pour laquelle elles travaillent principalement, sont aujourd'hui en train de vivre dramatiquement la crise du secteur ${ }^{12}$.

FiguRE 5

Volume d'affaires (en \% du chiffre d'affaires) pour les deux premiers donneurs d'ordres

(\%)

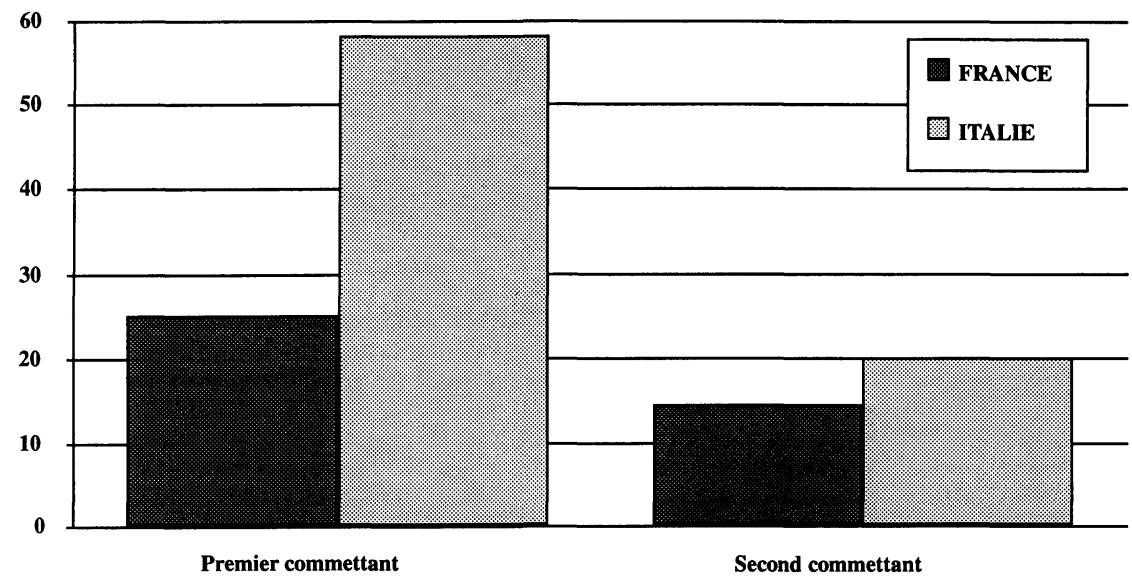

En conclusion, cette section met en évidence le fait que les diversités structurelles du secteur de l'aéronautique des deux pays analysés ont permis au système de sous-traitance aéronautique français de se développer avec plus d'une dizaine d'années d'avance par rapport au système italien. L'élément le plus significatif qui émerge est que les entreprises sous-traitantes françaises

12. À la suite de la crise du secteur de l'aéronautique, deux événements se sont vérifiés qui ont contribué à une réduction radicale du chiffre d'affaires des entreprises soustraitantes. Le premier est lié à la réduction de l'activité de sous-traitance due à la réduction du chiffre d'affaires global du secteur; le second est représenté par le retour dans les grandes entreprises de ces activités de sous-traitance internationale qui étaient auparavant, et pour une bonne part, décentrées sur les petites et moyennes entreprises sous-traitantes. 
ont une plus grande propension à l'exportation que les entreprises italiennes et un degré plus faible de dépendance aux entreprises «donneurs d'ordres».

Nous verrons dans la prochaine section que ces diversités n'influencent pas la structure du parc machines des entreprises sous-traitantes, mais qu'elles se manifestent par une conception et une diffusion différente du système qualité.

\section{Comparaison entre la sous-traitance italienne et française}

Après avoir mis en évidence quelques caractéristiques générales de la soustraitance en France et en Italie, cette partie sera dédiée à la comparaison entre les entreprises sous-traitantes des deux pays. On mettra particulièrement l'accent sur la diffusion des technologies informatisées (TI) et sur la gestion de la qualité dans les entreprises. On analysera le rôle joué par la formation sur la diffusion et l'implantation du système qualité dans les entreprises sous-traitantes.

\subsection{La composition du parc machines}

À l'intérieur du parc machines, la diffusion des nouvelles technologies assistées par ordinateur dans les entreprises sous-traitantes constitue un aspect important $^{13}$. En effet, les technologies assistées par ordinateur ont un impact positif sur la qualité puisqu'elles permettent de réduire les temps d'apprentissage, rendent plus stable le niveau qualitatif des produits et réduisent les temps de réponse aux commandes.

En outre, puisque les grandes entreprises ont été plus rapides dans l'introduction des technologies assistées par ordinateur (Cainarca et al., 1987), les entreprises sous-traitantes ont été obligées de suivre la tendance à long terme technologique pour éviter la perte de compétitivité et l'exclusion du rapport de sous-traitance. C'est pour cette raison que les technologies assistées par ordinateur ont représenté l'investissement principal des entreprises soustraitantes ces dix dernières années. La recherche sur le terrain confirme ce qui vient d'être énoncé ; elle met en évidence, en effet, la diffusion rapide des technologies assistées par ordinateur et montre que ce phénomène concerne aussi bien les entreprises sous-traitantes italiennes que françaises.

13. Par technologies informatisées, on entend ici l'ensemble des technologies de production et d'interface production-conception utilisées par les entreprises soustraitantes : machines-outils à contrôle numérique $(\mathrm{CN})$, centres d'usinage $(\mathrm{CU})$, systèmes de conception et d'interface conception-production (CAO, FAO et CFAO). 
En 1991-1992, environ $90 \%$ des entreprises sous-traitantes interrogées possédait au moins une machine-outil à contrôle numérique $(\mathrm{CN})$; ce pourcentage était de $60 \%$ en 1986-1987. Dans la même période, les entreprises qui utilisaient les centres de travaux (CdT) passaient de 26 à $66 \%$.

La diffusion des systèmes CAO, FAO et CFAO présente, elle aussi, une tendance positive. Le pourcentage d'entreprises utilisatrices de systèmes CAO passera de $29 \%$ en 1991-1992 à $67 \%$ en 1996-1997, avec une augmentation ultérieure de $50 \%$ par rapport à 1991-1992.

Du point de vue de la diffusion des nouvelles technologies assistées par ordinateur, il n'y a pas de différences significatives entre les entreprises soustraitantes italiennes et françaises. Même si au milieu des années 1980, les entreprises italiennes présentaient un léger retard, au début des années 1990, la situation apparaît plus ou moins homogène ${ }^{14}$.

Une innovation technologique croissante s'est imposée dans les entreprises sous-traitantes; elle se base sur la sélection des équipements les plus adaptés, flexibles et facilement intégrables dans le domaine de la fabrication. L'intérêt des entreprises se porte vers des technologies assistées par ordinateur capables de gérer de façon opportune le domaine de la production dans son ensemble, facilitant les opérations de transfert de données entre la zone de conception et celle de fabrication, permettant en même temps de réduire les temps de réponse aux commandes (Centre de travail à contrôle numérique et systèmes CFAO). La tendance des entreprises sous-traitantes est donc à l'intégration, que ce soit entre les différentes fonctions de l'entreprise à l'intérieur même de l'entreprise ou avec les donneurs d'ordres. Ce phénomène est homogène et concerne aussi bien les entreprises françaises qu'italiennes.

Il découle de ces dernières considérations qu'il n'existe pas de substantielles différences dans la composition du parc machines entre les entreprises sous-traitantes françaises et italiennes.

Toutefois, dans les pages qui suivront, on montrera qu'il existe de profondes différences dans le management qui déterminent l'orientation de l'entreprise envers la formation des ressources humaines et les problématiques de la qualité.

\subsection{La gestion de la qualité}

Nous focaliserons désormais sur la gestion de la qualité dans les entreprises sous-traitantes avec l'objectif de vérifier la diffusion de la gestion de la qualité

14. Pour chacune des technologies informatisées, le test de Fisher indique que les différences entre les entreprises italiennes et françaises ne sont pas statistiquement significatives. 
totale et de mettre en évidence les traits communs et les différences entre les deux réalités nationales.

La qualité est une des portes d'entrée les plus significatives dans le marché de la sous-traitance aéronautique. C'est pour cette raison que les entreprises sous-traitantes investissent de grandes ressources dans ce domaine. Deux éléments témoignent du vif intérêt de ces entreprises sous-traitantes du secteur de l'aéronautique pour la qualité. Le premier est l'incidence élevée du personnel qui se consacre à la qualité par rapport au total du personnel (en moyenne $7 \%$ environ, aussi bien dans les entreprises françaises que dans les entreprises italiennes ${ }^{15}$ ). Le second concerne l'importance hiérarchique du responsable de la qualité au sein des entreprises qui, en ligne avec la philosophie de la gestion de la qualité totale ${ }^{16}$, dépend du plus haut responsable de l'entreprise (le directeur général ou l'entrepreneur lui-même) et ce, dans toutes les entreprises sous-traitantes interrogées.

Le donneur d'ordres exerce un rôle d'orientation pour les entreprises sous-traitantes. En effet, l'introduction de la fonction qualité, dans les deux échantillonnages, est principalement motivée par la demande du commettant (figure 6).

FIGURE 6

Motivations de l'introduction de la fonction qualité

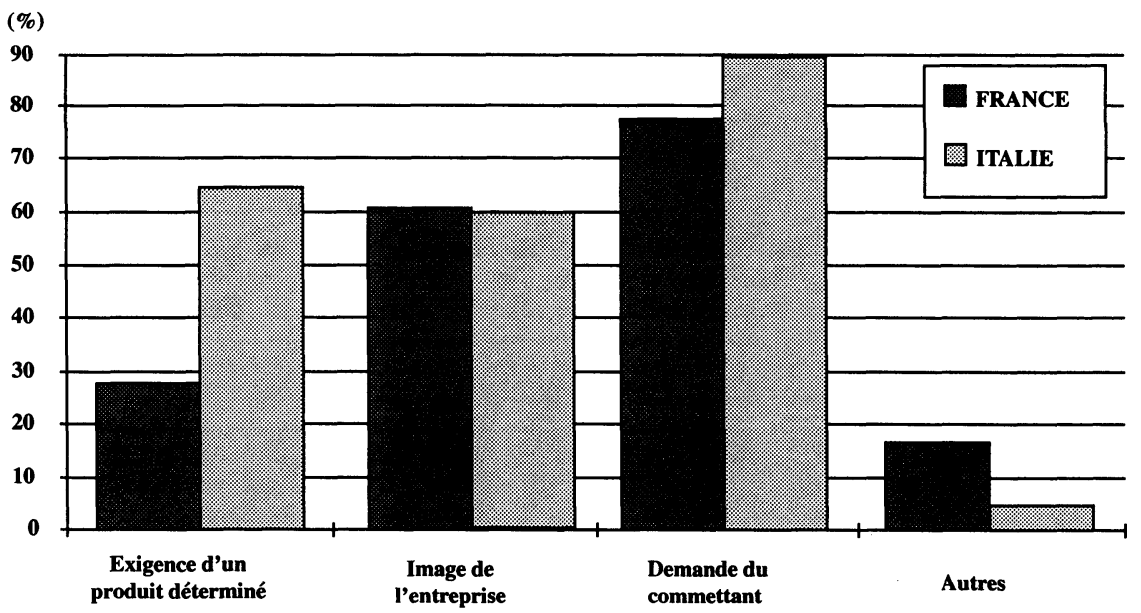

15. Ce pourcentage est supérieur à celui de nombreux autres secteurs. Par exemple, dans le domaine de la sous-traitance adressée au secteur de l'automobile, il est d'environ 4,6\% (Esposito et Raffa, 1994).

16. Selon la «gestion de la qualité totale », la qualité au sein de l'entreprise assume un rôle fondamental et ne doit pas dépendre hiérarchiquement des autres fonctions de l'entreprise. 
Ces pourcentages élevés indiquent que les commettants français et italiens poussent leurs sous-traitants à investir dans les ressources humaines et matérielles dans le domaine de la qualité et interviennent directement pour en guider le processus évolutif. Le rôle de guide de l'entreprise commettante se manifeste de façons diverses. Dans plus de $80 \%$ des rapports de soustraitance (figure 7), le commettant, par l'imposition de notes détaillées, provoque des changements de la fonction de qualité du sous-traitant en termes de ressources humaines et matérielles, et en termes de déroulement.

\section{FIGURE 7}

\section{Interventions du donneur d'ordre pour améliorer la qualité}

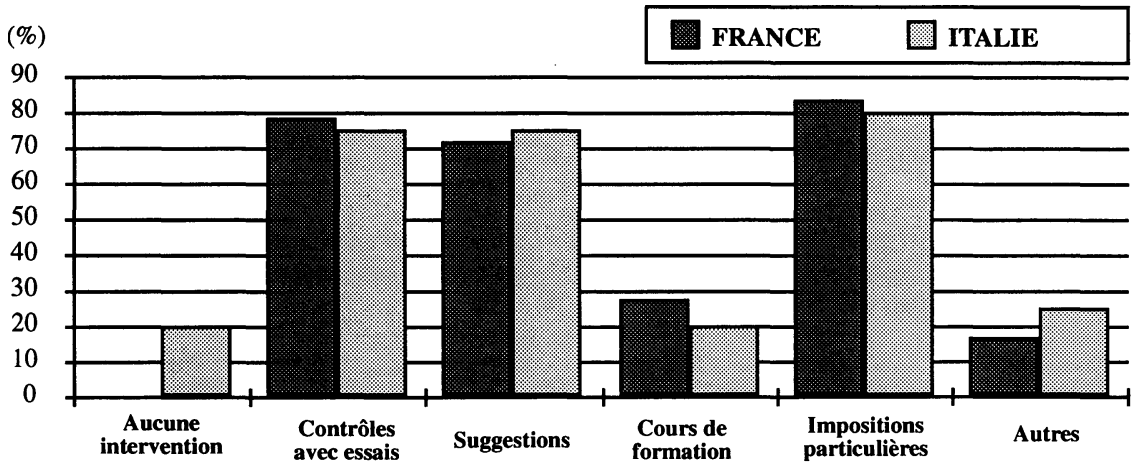

Dans 70 à $80 \%$ des rapports de sous-traitance, le commettant intervient directement pour modifier la fonction de qualité du sous-traitant par des suggestions, et dans 20 à $25 \%$ des cas, en fournissant des cours de formation aux responsables de la qualité des sous-traitants.

Par ces interventions, le commettant s'assure que les entreprises soustraitantes améliorent leur système de qualité et le modifient suivant ses indications. Cela détermine un processus d'approche du système de qualité du sous-traitant par le commettant.

Ce processus demande que les entreprises sous-traitantes répondent aux instances des commettants en investissant des ressources importantes dans le domaine de la qualité. Cette tendance est générale et se manifeste à mesure égale tant dans le cas des entreprises sous-traitantes italiennes que françaises. Toutefois, même s'il n'y a pas de différence entre les deux échantillonnages au regard des ressources investies dans le domaine de la qualité, de profondes divergences émergent si l'on considère la façon dont ces entreprises gèrent la qualité. 
Le cadre général sur les caractéristiques de la fonction qualité (figure 8) montre les différences principales entre les entreprises françaises et italiennes en ce qui concerne la gestion de la qualité :

- tandis que la majorité des entreprises françaises (plus de 70\%) formalisent les objectifs annuels de la qualité, seulement un petit nombre des entreprises italiennes (à peine $15 \%$ ) font de même ;

- plus de $70 \%$ des entreprises françaises développent des projets d'amélioration de la qualité, contre $30 \%$ des entreprises italiennes;

- même si la fonction qualité est considérée satisfaisante par $70 \%$ des entreprises italiennes et françaises, $90 \%$ des entreprises françaises considèrent cette fonction perfectible, contre $35 \%$ des entreprises italiennes ;

- toutes les entreprises françaises ont mis en place une activité de formation sur la qualité, contre $60 \%$ des entreprises italiennes.

Cela témoigne que, contrairement aux entreprises italiennes, les entreprises françaises sont orientées vers une philosophie de changement continu s'accordant avec celle de la gestion de la qualité totale.

FIGURE 8

\section{Caractéristiques de la fonction qualité}

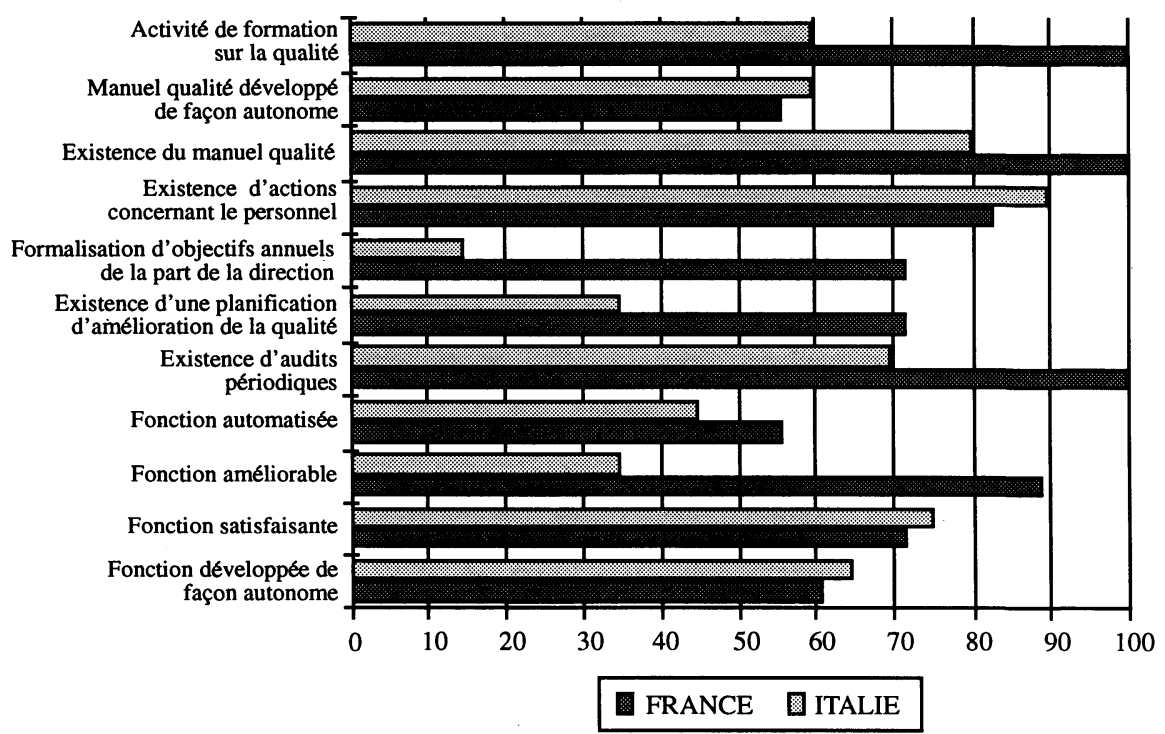

Revue internationale P.M.E., vol. 9, n 2, 1996 
Ce que l'on vient d'énoncer découle clairement des politiques des entreprises au sujet de la formation (figure 9). Dans les entreprises françaises, toutes les professions présentes dans l'entreprise sont concernées par les thématiques de la qualité, non seulement le domaine technique et productif $(100 \%$ des techniciens et $90 \%$ des ouvriers), mais aussi le domaine administratif (environ $90 \%$ des employés administratifs) et celui de la direction (environ $80 \%$ des cadres et des dirigeants de l'entreprise). Au contraire, dans les entreprises italiennes la formation a concerné presque essentiellement le secteur technique et productif ( $55 \%$ des techniciens et $50 \%$ des ouvriers). Le domaine administratif est peu touché (à peine $20 \%$ ), et encore moins le domaine de la direction (10\% des cadres et $15 \%$ des dirigeants).

Pour les entreprises italiennes, la qualité, apparemment, concerne essentiellement le domaine technique et productif, tandis que pour les entreprises françaises, elle concerne tous les domaines de l'entreprise, de la production à la direction.

FIGURE 9

\section{Professions concernées par la formation sur les thématiques de la qualité}

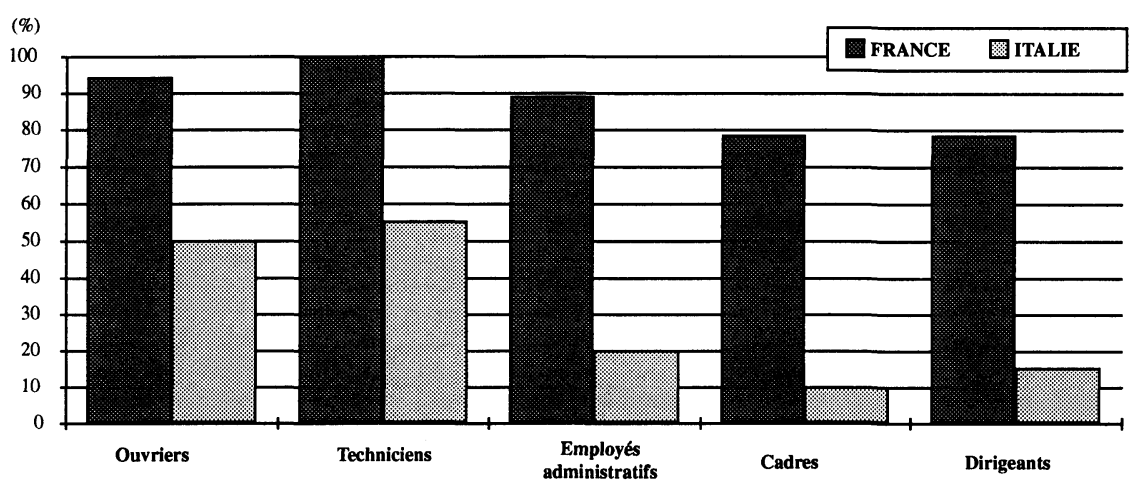

En résumé, les résultats de l'enquête sur le terrain montrent qu'on porte une grande attention aux problèmes de la qualité dans toutes les entreprises sous-traitantes analysées, et que cette attention se concrétise par de gros investissements en ressources humaines et matérielles. Toutefois, les entreprises italiennes se focalisent encore essentiellement sur le domaine de la production, signe évident d'une approche plus traditionnelle, attentive surtout au respect des indications techniques du commettant. Les entreprises françaises sont, au contraire, clairement orientées vers la gestion de la qualité totale. La qualité concerne tous les domaines de l'entreprise, de la production au domaine administratif, et ce, jusqu'à la direction. 


\section{Conclusion}

En Italie et en France, le système de la sous-traitance aéronautique est caractérisé par des entreprises de petites dimensions qui, en moyenne, comptent moins de 100 employés.

Les entreprises sous-traitantes des deux pays présentent une forte homogénéité en ce qui concerne les activités de production. On part des activités les plus traditionnelles, comme la mécanique de précision et le travail des tôles, aux activités qui caractérisent les nouveaux rapports de sous-traitance comme, par exemple, le travail des matériaux composites, l'assemblage spécialisé et la réalisation d'équipements.

Les entreprises sous-traitantes sont en train de développer une série d'innovations technologiques basées sur les technologies assistées par ordinateur qui sont plus flexibles et facilement intégrables à celles du donneur d'ordres. Ce phénomène est homogène et concerne également les entreprises italiennes et françaises. L'important investissement dans le domaine de la production a fait en sorte qu'au début des années 1990, aucune différence significative n'ait pu être relevée entre les entreprises françaises et italiennes en ce qui concerne le parc machines.

Les entreprises sous-traitantes italiennes et françaises du secteur de l'aéronautique présentent donc de fortes analogies au regard de la dimension, des activités de production et de la structure du parc machines.

Toutefois, ces entreprises présentent d'importantes différences dans leurs rapports avec les donneur d'ordres et dans leur façon de gérer les problèmes de la qualité. Cela est relié, d'une part, aux dissemblances dans l'histoire des entreprises aéronautiques italiennes et françaises et, d'autre part, au rôle inégal que jouent ces pays dans l'oligopole mondial de ce secteur.

L'industrie aéronautique française a développé son réseau d'entreprises sous-traitantes avec plus d'une décennie d'avance par rapport à l'industrie italienne.

Les entreprises sous-traitantes françaises, par rapport aux italiennes, montrent une propension majeure à l'exportation et une moindre dépendance envers les entreprises "donneurs d'ordres». Par contre, les entreprises italiennes sont très liées aux commandes nationales et focalisent leurs activités sur les deux premiers donneurs d'ordres. Ce fait devient un point faible important puisque dans les périodes de récession, les entreprises sous-traitantes italiennes sont condamnées à subir la crise du secteur et du donneur d'ordres. Au contraire, les entreprises françaises qui opèrent avec plusieurs donneurs d'ordres, mais aussi dans plusieurs secteurs, peuvent s'orienter plus facilement vers des donneurs d'ordres et des secteurs qui souffrent moins de la récession. 
Les entreprises italiennes peuvent tirer une leçon de l'expérience française : il est possible d'instaurer des rapports étroits de collaboration avec les donneurs d'ordres principaux tout en diversifiant le chiffre d'affaires entre plusieurs entreprises «donneurs d'ordres ». Cette stratégie a une conséquence importante : elle amoindrit la dépendance des sous-traitants aux donneurs d'ordres et permet aux entreprises sous-traitantes de mieux réagir durant les phases de récession économique.

L'élément qui différencie davantage la réalité des entreprises françaises et italiennes, et qui mérite donc une attention toute particulière, concerne leurs façons de traiter la question de la qualité.

Les résultats de l'enquête sur le terrain montrent que dans toutes les entreprises françaises et italiennes, l'attention que l'on porte aux problématiques de la qualité est forte et c'est dans ce domaine qu'elles réalisent d'importants investissements en termes de ressources humaines et matérielles. Toutefois, les entreprises italiennes focalisent encore essentiellement sur le domaine de la production, signe évident d'une approche de type plus traditionnel, donc surtout attentives au respect des indications techniques fournies par le donneur d'ordres. Les entreprises françaises sont au contraire clairement orientées vers la gestion de la qualité totale et cette qualité concerne tous les domaines de l'entreprise, de la production au domaine administratif, jusqu'à la direction.

Ce décalage dans les orientations vers la qualité peut se traduire, à long terme, par une perte de compétitivité des entreprises italiennes. Nous retenons donc que le problème de l'orientation stratégique vers la qualité constitue un important sujet de réflexion pour le management des entreprises italiennes. Dans cette optique, les résultats de la recherche nous amènent à dégager quelques actions prioritaires qui peuvent être résumées en cinq points.

1. Réduire la part du chiffre d'affaires du premier donneur d'ordres tout en diversifiant vers des donneurs d'ordres provenant d'autres secteurs de haute technologie. Ce ne sont pas les machines qui font la différence avec les entreprises françaises, mais la philosophie d'entreprise et la gestion.

2. Viser la création de consortium : une stratégie de collaboration et de coopération entre les entreprises sous-traitantes est indispensable ; cette stratégie doit viser la présentation des entreprises sous-traitantes italiennes sur la scène internationale et européenne.

3. Mettre en place un changement dans la philosophie de l'entreprise : dépasser la phase de la grande entreprise-atelier gérée par le propriétaire ou l'entrepreneur. 
4. Développer un système de qualité totale. La qualité devient un levier stratégique. Ce n'est donc pas une exigence du donneur d'ordres (on réalise des manuels pour satisfaire les exigences du donneur d'ordres qui ne sont jamais utilisés), mais un levier stratégique de l'entreprise qui doit opérer avec plusieurs donneurs d'ordres, dans plusieurs secteurs et à l'échelle internationale.

5. La formation des ressources humaines devient fondamentale.

\section{Bibliographie}

Altersohn, C. (1992), De la sous-traitance au partenariat industriel, Paris, L'Harmattan.

AOKI, M. (1988), Information, Incentives, and Bargaining in the Japanese Economy, New York, Cambridge University Press.

AOKI, M. (1984), The Cooperative Game Theory of the Firm, Londres, Oxford University Press.

ASANUMA, B. (1989), «Manufacturer supply relationships in Japan and the concept of relation-specific skill», Journal of the Japanese and International Economies, $\mathrm{n}^{0} 3$.

Barthomieu, C., C. Chanel-Reynaud, J.P. Guighard, A. Hanaut et A. Longhi (1983), Structure industrielle et sous-traitance, Paris, Presses universitaires de France.

Bodin, B. (1963), «Préface», dans A. Sallez et J. Schlegel, La sous-traitance dans l'industrie. Modalités, lacunes, prospectives, Paris, Dunod.

CainarCa, G.C., M.G. Colombo et S. MARIOTTI (1987), «Innovazione e diffusione : il caso dell'automazione flessibile », L'Industria, $\mathrm{n}^{0} 4$.

CEE (1993), The European Space Industry. Trading Position and Figures, Commission économique des Communautés européennes, Bruxelles.

ChaIllou, B. (1977), « Définition et typologie de la sous-traitance», Revue économique, $\mathrm{n}^{0} 2$.

COASE, R.H. (1937), «The nature of the firm», Economica, novembre, p. 386-405.

Colombo, M. et S. MARIOTTI (1994), «L'eccellenza nelle relazioni cliente fornitore. Osservazioni sul modello giapponese », Economia \& Management, Luglio.

DAVID, A.J. (1990), «The customer/supplier relationship. The Nissan way », Total Quality Management, vol. 1, $\mathrm{n}^{0} 10$.

De MaIo, A. et E. MAggiore (a cura di) (1992), Organizzare per innovare. Rapporti evolutivi cliente-fornitore, Milan, ETASLibri. 
Del MonTE, A. (1991a), «Alcuni modelli di interpretazione nei rapporti fra grandi e piccole imprese», Convegno Rapporti di collaborazione tra grandi e piccole imprese, mai, Naples.

Del MonTE, A. (1991b), Introduzione ai lavori del convegno « Recenti contributi alla teoria dell'impresa », 16-17 mai, Naples, Facoltà di Economia e Commercio, Università di Napoli.

EsPosito, E. et C. STORTo (1992), «Qualitative and structural changes of the subcontracting firms : a micro-analytical approach to the study of interfirms relationships », EIASM, Research in Entrepreneurship 6th Workshop, 26-27 novembre, Barcelone.

EsPosito, E. et M. RAFFA (1994), «L'evoluzione del sistema della subfornitura nell'industria italiana », Economia \& Management, Luglio.

ESPOSITO, E. et M. RAFFA (1993), «The evolution of sub-contracting firms. Empirical evidence», Comptes rendus du $23^{e}$ European Small Business Seminar, 15-17 septembre, Irlande du Nord, vol. 2, p. 840-858.

EsPosito, E. et M. RAFFA (1991), « Supply in Hi-Tech industry : the role of the small businesses », Atti della 36th ICSB World Conference, 24-26 juin, Vienne.

FERRAND, P.M. (1984), Subfornitura e approvvigionamenti nell'evoluzione del sistema aziendale, Franco Angeli, Milan.

FERRATA, R. et S. MEREgalli (1993), «Ipotesi di comportamenti strategici caratterizzati dalla presenza dell' information technology », Economia \& Management, Luglio.

FLETCHER, D. (1993), « Strategic alliances and value adding networks : a critical review », RENT VII (Research in Entrepreneurship Conference), 26-27 novembre, Budapest, Hongrie.

GRANDI, A., G.L. MARZOCCHI et A. ZANONI (1994), «Verso l'organizzazione a rete. Il ruolo dell'IT », Economia \& Management, Luglio.

Halén, L., J. Johanson, et N. SEYed-Mohamed (1991), «Interfirm adaptation in business relationships », Journal of Marketing, vol. 55, avril.

Hines, P. (1994), Creating World Class Suppliers, Unlocking Mutual Competitive Advantage, Londres, Pitman Publishing.

IMRIE, R. et J. MORRIS (1992), «A review of recent changes in buyer-supplier relations », Omega, vol. 20, $\mathrm{n}^{\text {os }}$ 5-6.

IMRIE, R. et J. MORRIS (1993), «Japanese style subcontracting. Its impact on European industries », Long Range Planning, vol. 26, $\mathrm{n}^{\circ} 4$.

JANE'S (1994), Jane's all the World Aircraft, Londres. 
KLEIN, B., R. CRAWFORD et A. AlChIAN (1978), «Vertical integration, appropriable rents and the competitive contracting process », The Journal of Law and Economics, $\mathrm{n}^{\mathrm{0}} 21$.

LAmming, R. (1993), Beyond Partnership, Strategies for Innovation and Lean Supply, New York, Prentice-Hall.

LAWTON-SMITH, H., K. DiCKSON et S. LlOYD-SMITH (1991), «There are two sides to every story : innovation and collaboration within networks of large and small firms », Research Policy, ${ }^{\circ} 20$.

LECLER, Y. (1992), «L'avenir du partenariat à la japonaise», Revue Francaise de Gestion, novembre-décembre.

LEIBENSTEI, H. (1966), "Allocative efficiency versus X-efficiency », American Economic Review, n ${ }^{0}$ 56, p. 383-415.

MERLI, G. (1991), Total Quality Management, Turin, Isedi.

Minato, T. (1992), «Strategic alliance between small and large business », Première conférence de la Fédération internationale des associations universitaires en management (IFSAM), Tokyo, 7-9 septembre.

MITI (1991), White Paper on Small and Medium Enterprises in Japan.

MOREAU, M. (1985), « Structures et situation actuelle et future de l'industrie aéronautique et spatiale francaise », Revue d'Économie Industrielle, $\mathrm{n}^{0} 31$, p. 177-184.

MORRIS, J. (1992), «Organization in an alien environment : Japanese style subcontracting relations in the U.K. », Première conférence de la Fédération internationale des associations universitaires en management (IFSAM), Tokyo, 7-9 septembre.

MOWERY, D. (1991), "International collaboration in the commercial aircraft industry », dans L.K. Mytelka (éd.), Strategic Partnership - States, Firms and International Competition, Rutherford, Farleigh Dickinson University Press.

OCDE (1991), Science and Technology Indicators, Paris.

PERrouX, F. (1966), L'Économie du XX'e Siècle, (traduction italienne) L'economia del XX secolo, Milan, Etas Kompass.

RAFFA, M. (1992), « Strategie di cooperazione : l'evoluzione della subfornitura nei settori a tecnologia sistemica», dans R. Filippini, G. Pagliarani et G. Petroni (éd.), Progettare e gestire l'impresa innovativa, Milan, ETASLibri.

SAGET, F. (1988), «Partnership between small and large firms : what does it entail ? How is it developing ? ", dans Commission économique des Communautés européennes, Partnership Between Small and Large Firms, Londres, Graham \& Trotman.

SAKO, M. (1988), «Partnership between small and large firms : the case of Japan », dans Commission économique des Communautés européennes, Partnership Between Small and Large Firms, Londres, Graham \& Trotman. 
SAKo, M. (1992), Price, Quality and Trust. Inter-firm Relations in Britain \& Japan, Cambridge University Press.

SALlEZ, A. (1977), « De l'analyse structurelle de la firme à la division spatiale du travail », Économie appliquée, $\mathrm{n}^{0} 2$.

Sallez, A. et J. SChlegel (1963), La sous-traitance dans l'industrie. Modalités, lacunes, prospectives, Paris, Dunod.

SALLEZ, A. (1975), « Sous-traitance, productivité économique et croissance régionale », Économie appliquée, $\mathrm{n}^{\text {os }} 2-3$.

SAMSON, R. (1993), «Competing with the Japanese strategies for European business », Long Range Planning, vol. 26, $\mathrm{n}^{0} 4$.

SCOTT, C.S. et R. WeStbRooK (1991), «New strategic tools for supply chain management », International Journal of Physical Distribution \& Logistics Management, $\mathrm{n}^{0} 1$.

TEECE, D.J. (1992), « Competition, cooperation, and innovation - Organizational arrangements for regimes of rapid technological progress », Journal of Economic Behavior and Organization, $\mathrm{n}^{\circ} 18$.

The Technology Atlas Team (1987), «Components of technology for resources transformation », Technological Forecasting and Social Change, vol. 32, $\mathrm{n}^{\circ} 1$.

Tyson, L. (1992), Who's Bashing Whom? Trade Conflict in High Technology Industries, Institute for International Economics, Washington, D.C.

VENNIN, B. (1975), «Pratiques et signification de la sous-traitance dans l'industrie automobile en France », Revue économique, $\mathrm{n}^{0} 2$.

Williamson, O.E. (1975), Markets and Hierarchies: Analysis and Antitrust Implication, New York, The Free Press.

Williamson, O.E. (1979), «Transaction-cost economics: the governance of contractual relations ", The Journal of Law and Economics, $\mathrm{n}^{\circ} 2$.

Williamson, O.E. (1985), The Economic Institutions of Capitalism, New York, The Free Press. 